\title{
How May I Help You? - State of the Art and Open Research Questions for Chatbots at the Digital Workplace
}

\author{
Raphael Meyer von Wolff \\ University of Goettingen \\ rmvw@uni-goettingen.de
}

\author{
Sebastian Hobert \\ University of Goettingen \\ shobert@uni-goettingen.de
}

\author{
Matthias Schumann \\ University of Goettingen \\ mschuma1@uni-goettingen.de
}

\begin{abstract}
Chatbots become quite hyped in recent times as they can provide an intuitive and easy-to-use natural language human-computer interface. Nevertheless, they are not yet widespread in enterprises. Corresponding application areas for collaboration at digital workplaces are lacking and prior research contributions on this topic are limited. In this research paper, we aim at surveying the state of the art as well as showing future research topics. Thus, we conducted a structured literature review and showed that only few first research contributions exist. We also outline current potentials and objectives of chatbot applications. In the discussion of the results of our structured literature review, we show that research gaps are present. To tackle the research gaps, we derive open research questions.
\end{abstract}

\section{Introduction}

In recent years, a growing digitalization of the economy can be observed. In particular, this influences enterprises and the way how employees work at office workplaces. Based on this increasing use of innovative technologies, the workplace of the future turns into a digital-enhanced workplace [33, 35]. Established paperbased working practices vanish and new forms of collaboration as well as office and working structures are spreading. Employees demand the use of new technologies at the workplace that they know from private use [34, 35, 40, 65]. In addition, a second "megatrend" should be considered: the distribution of messaging-services for communication and collaboration among employees in the day-to-day business [22]. This influence of location- and device-independent communication also effects and shapes the digital workplace. Despite the advantages of using innovative technologies, this results in an increasing number of communication channels and corresponding information sources. Employees also tend to use multiple information systems in their day-to-day business simultaneously, which leads to an application overload. Thus, employees spend an increasing amount of time for searching, editing or sharing of information [52, 65], which could further affect the employees' productivity in their work tasks negatively $[12,38]$.

To address these problems, it is necessary to filter information to avoid information overloads as well as to reduce the workload during daily tasks by providing appropriate assistance. One promising technology for this is the use of artificial intelligence in the form of chatbots. They provide a human-computer interface using natural language-based dialogs and are capable of assisting or automating tasks as well as filtering and providing information $[20,54,55]$. Although practitioners assume that chatbots can influence employees' productivity positively, the technology itself is still in an early development stage. Even though dialog-based systems (e.g., Amazon's Alexa or Ikea's Anna) are popular among consumers, chatbots are currently not yet widespread in enterprises and corresponding application areas are lacking $[10,36]$. This is also reflected in the scientific knowledge base, as prior research is limited in this infancy research area. Therefore, we aim at providing an in-depth analysis of the current state of the art as an entry point for future research [24]. Based on a structured literature review, we analyze the current literature, describe the outcomes and postulate open research questions. Thus, we ask the following research questions:

RQ1: How can the state of the art of chatbots at the digital workplaces be systematized?

RQ2: Which research questions exist in the research area that have not been answered yet?

To answer these questions, the remainder is structured as follows: Below, we present the theoretical foundations in section 2 and describe the methodical approach of our literature review in section 3. In section 4 , we outline the results of our literature review, discuss them in section 5 and postulate open research questions in section 6. We summarize our findings in section 7. 


\section{Theoretical Foundations}

\subsection{Digital Workplace}

The design of workplaces has a long tradition in human-oriented computer science. In recent years, the nature of work has been changed and affected enterprise technologies as well. New technologies emerged and todays work becomes more digitally. Furthermore smart systems replaced traditional human capabilities and are used to perform mainly routine tasks [51, 65]. However, the information access is still a major problem at the workplace. Thus, new technologies are needed to address this problem in the future [65]. As mentioned by [51], future application systems have to be user-centric, allow transforming work practices and must provide flexibility. Therefore, it is necessary for enterprises and especially at workplaces to process information in the appropriate manner to reduce uncertainty and equivocality in daily work. In addition to that, scientific theories also provide insights about how to address these problems: For instance, the media richness theory gives indications how information access should be designed [17]. To enable research for chatbots at digital workplaces, it is necessary to define the application area in a first step. In general, a digital workplace is not limited to a physical place. Instead it is a virtual summary of tasks on information, e.g., searching, transforming, documenting [8]. Nowadays, this is also known as knowledge work [65]. Considering today's focus on application systems and messaging services, the digital workplace is usually location-independent, sometimes mobile and often integrates different technologies, people and processes $[40,65]$.

Thus, $a$ digital workplace combines (IT)technologies, processes and people for information processing in and between enterprises. Therefore, the focus lies on working with information and includes a high relevance of communication and collaboration among the involved people and/or application systems.

Based on this, the following characteristics are noteworthy: First, the primary focus of the digital workplace is the use of information systems for daily tasks and requires an increasing utilization of information for the task fulfillment. In this way, we differentiate it from physical work (e.g., production processes or maintenance tasks, as focused in [31]). Therefore, it is firstly necessary to collect or share information that are required for the task execution or to solve (novel) problems. Secondly, employees have to work collaboratively. Therefore, they need systems to support the teamwork. Thirdly, employees have to learn continuously for example to adapt to changes in work scenarios. To take these characteristics into account, it is necessary to put human work practices and their context in the center when investigating the potential of digital technologies like chatbots [51].

\subsection{Chatbots}

Since the first chatbots ELIZA [64] and ALICE [62] different approaches for conversational information systems were pursued, but the main characteristics have remained largely the same: A chatbot is a special kind of an application system, whose functions are accessible via a dialog-based user interface, e.g., through messaging services [39]. It uses artificial intelligence technologies to provide a natural language user interface to various databases or APIs for the execution of work tasks. Thus, users can communicate - by text or audio in a natural and intuitive way with application systems $[1,2,12,30,41]$.

Thus, a chatbot is an application system that provides a natural language user interface for the human-computer-integration. It usually uses artificial intelligence and integrates multiple (enterprise) data sources (like databases or applications) to automate tasks or assist users in their (work) activities.

Additional characteristics of chatbots are: First, chatbots can perform actions reactively, proactively as well as autonomously based on user inputs or changes in the environment. Second, chatbots are adaptive and capable of self-learning to handle context information or consider user preferences in future dialogs.

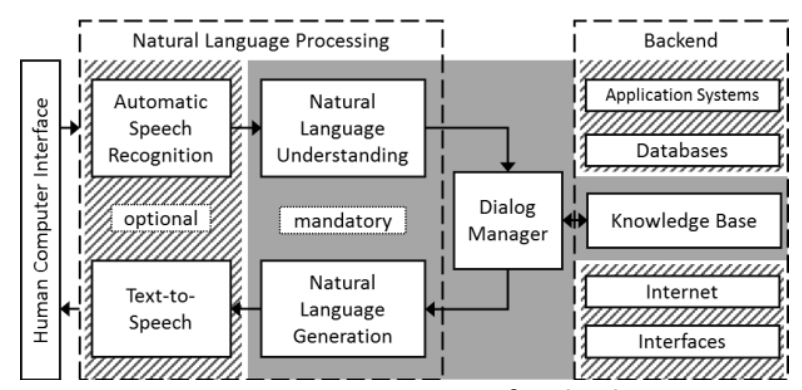

Figure 1. Components of a chatbot

The technical architecture of a chatbot consists of four mandatory and few optional modules $[9,41]$ (see Figure 1). As input, voice or text is possible. If voice is chosen, it has to be processed by automatic speech recognition to get machine-readable text. Afterwards, the natural language understanding analyses the input, dismantles it as well as examines it for patterns. Then the dialog manager processes the outcome against the backend and enquiries the data or knowledge bases, executes application systems or calls an API. After processing the user input, the results will be transformed in natural language, e.g., questions or simple answers, via a natural language generation module. Finally, the 


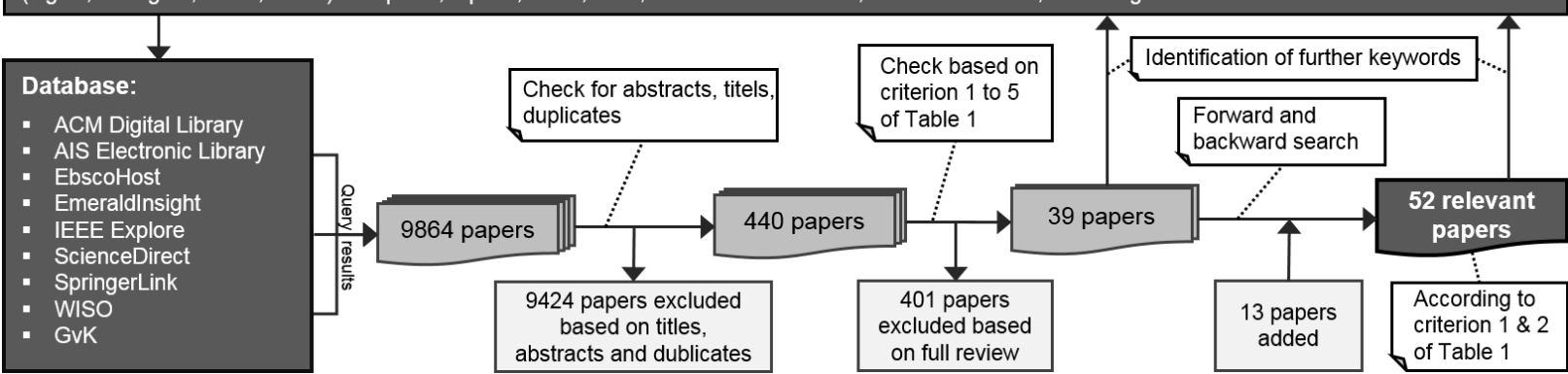

Figure 2. Research framework

generated text can be outputted as audio by the text-tospeech component. For this research study, we focus on the combination of the mentioned components as an entire application system and not on the design of specific technical components. In particular, we analyze the use of chatbots for supporting the daily work of single employees at the digital workplace as described in 2.1. In other words, we understand chatbots as a new medium for human-computer interaction [20].

\section{Methodical approach}

To assess the state of the art and to answer our research questions, we conducted a structured literature review [16, 19, 63]. Hereto, we examined current research approaches and application domains and potentials as well as objectives of using chatbots at digital workplaces. According to the aim, we intended to accumulate an almost complete census of relevant literature. Therefore, we used accessible search results of scientific databases as the basis for our data collection. To perform the search, we used English and equivalent German keywords (Figure 2; see the online appendix at http://bit.ly/ChatbotsatWorkplaces for a detailed overview of the used search strings). Before including a paper in our literature analysis, we checked the quality of the identified papers as follows: We included only reviewed and published scientific papers to reach a proper level of quality. Additionally, we took into account that papers provide completed research studies with comprehensible results and cite an adequate number of references. In addition to scientific research publications, we added published practice literature that reflect the current state of the art in enterprises. By doing this, we aim at transferring the results from practice in order to harness them in scientific. The search period was not limited, but we took care that relevant papers comply with the actual state of technology. As we finished the data collection in early 2018, we included literature published until end of 2017.
The found papers were filtered as follows [23]: Firstly, we checked the titles and abstracts of all query results and excluded duplicates. Secondly, we reviewed the content of the remaining papers in detail. Therefore, we predefined a list of criteria, based on our research goal, to classify literature as relevant (see Table 1). As shown, only the first two criteria represent relevant literature for our review. We used criteria 3-5 for excluding irrelevant literature.

After this initial search process, we conducted a forward and backward search and added 13 papers. Overall, we identified 52 relevant research papers, which we analyzed for further study to figure out the contributions, application areas and objectives of chatbots at digital workplaces (see Figure 2).

Table 1. Criteria of relevant papers

\begin{tabular}{|c|l|}
\hline Criteria & Description \\
\hline 1 & $\begin{array}{l}\text { Relevant are articles that examine chatbots and } \\
\text { application areas at the digital workplace jointly. }\end{array}$ \\
\hline 2 & $\begin{array}{l}\text { Relevant are articles that examine chatbots in } \\
\text { non-work-related application areas only if the } \\
\text { targeted use cases also exists in a comparable } \\
\text { way at the digital workplace (e.g., information } \\
\text { search tasks or online shopping). }\end{array}$ \\
\hline 3 & $\begin{array}{l}\text { Irrelevant are articles that examine chatbots in } \\
\text { general but in non-transferable application areas } \\
\text { for the digital workplace. }\end{array}$ \\
\hline 4 & $\begin{array}{l}\text { Irrelevant are articles that examine digital (office) } \\
\text { workplaces without being responsive to chatbots } \\
\text { or natural language assistance systems. }\end{array}$ \\
\hline 5 & $\begin{array}{l}\text { Irrelevant are articles that examine only technical } \\
\text { aspects or single components of chatbots, e.g. } \\
\text { mathematical algorithms or interface designs. }\end{array}$ \\
\hline
\end{tabular}

\section{Results}

In the following, we describe the results of our literature review. First, we present some descriptive findings. Afterwards, we outline the research contributions, potentials as well as objectives of using 
Table 2. Classification of identified relevant literature

\begin{tabular}{|c|c|c|c|c|c|c|c|c|c|c|c|c|c|c|c|c|c|c|c|c|c|c|c|c|c|c|c|c|c|c|}
\hline \multirow{2}{*}{\multicolumn{2}{|c|}{ Paper }} & \multicolumn{6}{|c|}{ Research contribution } & \multicolumn{17}{|c|}{ Potential } & \multicolumn{6}{|c|}{ Objective } \\
\hline & & $\mathrm{CS}$ & IA & ET & SS & $\mathrm{CW}$ & WF & $\stackrel{-1}{a}$ & $\overrightarrow{1}$ & $\stackrel{0}{a}$ & $\stackrel{m}{a}$ & में & $\stackrel{\sim}{a}$ & $\stackrel{0}{a}$ & $\stackrel{\overrightarrow{1}}{\mathrm{a}}$ & $\stackrel{\infty}{\stackrel{1}{a}}$ & $\Sigma$ & $\vec{N}$ & N & $\stackrel{m}{\Sigma}$ & ज & $\stackrel{\sim}{\sim}$ & $\ddot{N}$ & $m$ & 다 & กี & m & O & เก & ర̊ \\
\hline \multirow{9}{*}{ 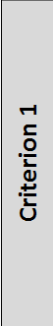 } & [10] & $\bullet$ & & & & & & & & $\bullet$ & & & & & & & $\bullet$ & & & $\bullet$ & & & & & & & & • & & $\bullet$ \\
\hline & [15] & $\bullet$ & & & & & & & & $\bullet$ & & & & & & & & & & & $\bullet$ & & & & & & & & $\bullet$ & \\
\hline & [18] & & & & $\bullet$ & & & & & $\bullet$ & & & & & & & & & $\bullet$ & & $\bullet$ & & & & $\bullet$ & & & & & $\bullet$ \\
\hline & [27] & & & & $\bullet$ & & & & $\bullet$ & $\bullet$ & & & & & & & & & $\bullet$ & & & $\bullet$ & & & $\bullet$ & & & & & $\bullet$ \\
\hline & [28] & & & $\bullet$ & & & & $\bullet$ & $\bullet$ & & & & & & & & & & & & & & $\bullet$ & $\bullet$ & $\bullet$ & & & & & $\bullet$ \\
\hline & [30] & & & & $\bullet$ & & & & & $\bullet$ & & & & & & & $\bullet$ & $\bullet$ & $\bullet$ & & & $\bullet$ & & & $\bullet$ & & & & & $\bullet$ \\
\hline & [38] & & & & & $\bullet$ & & $\bullet$ & & & & & & & & & & & & & & $\bullet$ & $\bullet$ & $\bullet$ & $\bullet$ & $\bullet$ & & & & \\
\hline & [54] & & & & & & $\bullet$ & & & & & & & & & & $\bullet$ & & $\bullet$ & & & & & & $\bullet$ & $\bullet$ & $\bullet$ & $\bullet$ & & $\bullet$ \\
\hline & [59] & $\bullet$ & & & & & & & & $\bullet$ & & & & & & & & & $\bullet$ & & & & & & $\bullet$ & & & & & $\bullet$ \\
\hline \multicolumn{2}{|c|}{ Sum criterion 2} & 11 & 11 & 2 & 0 & 0 & 19 & 20 & 10 & 8 & 1 & 8 & 5 & 3 & 1 & 1 & 3 & 14 & 3 & 0 & 3 & 6 & 4 & 7 & 40 & 4 & 7 & 6 & 6 & 11 \\
\hline \multicolumn{2}{|c|}{ Sum Total } & 14 & 11 & 3 & 3 & 1 & 20 & & & & & 43 & & & & & & & & 27 & & & & 9 & 47 & 6 & 8 & 8 & 7 & 18 \\
\hline \multicolumn{2}{|c|}{ Legend: } & \multicolumn{6}{|c|}{$\begin{array}{l}\text { CS Customer support } \\
\text { IA Information acquisition } \\
\text { ET Education and training } \\
\text { SS Self-service } \\
\text { CW Collaborative work } \\
\text { WF Without focus }\end{array}$} & \multicolumn{9}{|c|}{\begin{tabular}{|l} 
P1 Information search tasks \\
P11 Request of FAQs \\
P12 Answer customer questions \\
P13 Answer employee questions \\
P14 Searching for products \\
P15 Query weather information \\
P16 Query traffic information \\
P17 Query of tasks and appointments \\
P18 Provide maintenance information
\end{tabular}} & $\begin{array}{r}\text { P2 St } \\
\text { P21 } \\
\text { P22 } \\
\text { P23 } \\
\text { P24 } \\
\text { P25 } \\
\text { P26 } \\
\text { P3 Te }\end{array}$ & $\begin{array}{l}\text { andard } \\
\text { Shopp } \\
\text { Emplo } \\
\text { Custon } \\
\text { Bankin } \\
\text { Arrang } \\
\text { Setting } \\
\text { aching }\end{array}$ & $\begin{array}{l}\text { ized } r \\
\text { ng } \\
\text { eee se } \\
\text { ner se } \\
\text { g } \\
\text { e app } \\
\text { up re } \\
\text { and I }\end{array}$ & $\begin{array}{l}\text { outine } \\
\text { If-serv } \\
\text { If-serv } \\
\text { ointminde } \\
\text { earnin }\end{array}$ & $\begin{array}{l}\text { proce } \\
\text { ice } \\
\text { ice } \\
\text { ints an } \\
\text { rs } \\
\text { tasks }\end{array}$ & $\begin{array}{l}\text { esses } \\
\text { nd me }\end{array}$ & tings & & \multicolumn{6}{|c|}{$\begin{array}{l}\text { O1 Natural language user interface to } \\
\text { information systems } \\
\text { O2 Uniformly, device-independent and } \\
\text { mobile access to application systems } \\
\text { O3 Increase efficiency and productivity } \\
\text { O4 Decrease of time effort } \\
\text { O5 Reduce of costs } \\
\text { O6 Relieve employees by take over / } \\
\text { automate tasks }\end{array}$} \\
\hline
\end{tabular}

chatbots at the digital workplace. Due to the extent, we focus on the main contributions of the analyzed papers by summarizing the results briefly. The full overview of the relevant papers, their categorizations and some descriptive statistical analysis are available in an online appendix: http://bit.ly/ChatbotsatWorkplaces.

\subsection{Descriptive results}

The analysis of our relevance criteria clearly shows that most of the articles correspond to criterion 2 of Table 1 (see Figure 3). Only 9 out of 52 articles examine the application of chatbots at the digital workplace. Due to this limited amount of relevant literature, it is essential to include articles matching criterion 2. Thus, we examine the results of closely related articles that did not mention digital workplaces directly.

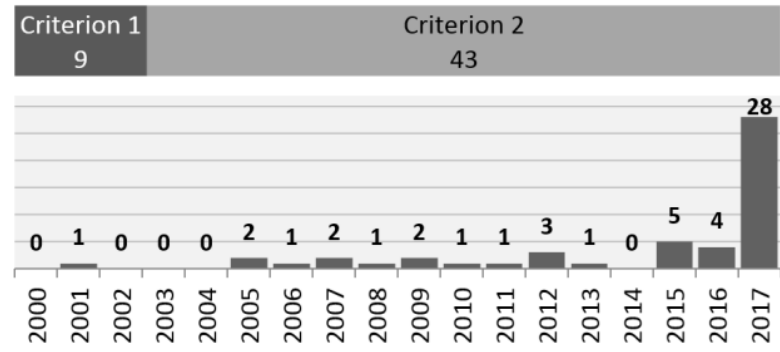

Figure 3. Descriptive distributions

The distribution by publication year (see Figure 3) clearly shows the relevance for the topic as already described in the introduction. Considering publications until 2016, we identified only few relevant papers per year. In 2017, the number of publications increased significantly. In addition, two articles could already be found online-first and were added to 2017.

\subsection{Application domains}

Our first research goal was to identify and categorize the application domains focused in the actual research. Therefore, we aimed at identifying research contributions belonging to the application domains of chatbots in office work tasks and categorized the articles based on that. This resulted in six categories of application domains at the workplace (see Table 2 and Figure 4) which are further subdivided by the art of the paper (scientific or practice-oriented).

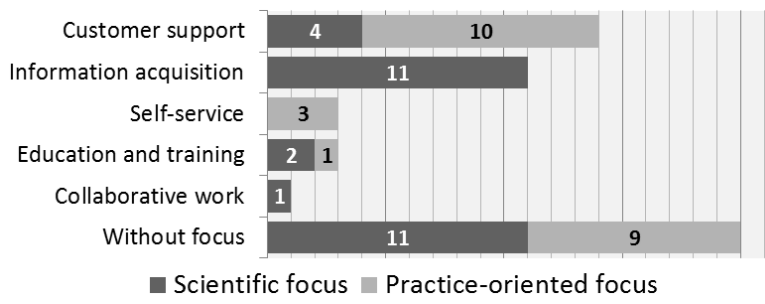

Figure 4. Categorization of application domains

As shown in Figure 4, the scientific research focuses mainly on information acquisition. The practiceoriented literature on the other side mostly focuses on customer support. Notably is that we identified a large amount of - scientific as well as practice-oriented papers without a specific research focus. Just a few authors mentioned the topics self-service, education and training, and especially collaborative work, which are all typical office work tasks. Summarizing, we can already detect a literature gap belonging the use of chatbots for collaboration and digital office work.

Specifically, most of the papers address the field of customer support [13], e.g., develop a chatbot for the information acquisition for products or services. The 
findings show that users can get needed information with a lower amount of clicks. [4] describe an ontologybased chatbot for the same area. Based on the ontology, the maintenance effort is reduced. A dynamic approach is followed by [14, 15]. Their chatbot is capable of creating a dynamic goal fulfillment map to answer requests. Evaluations show, that the resulting chatbot is able to handle longer conversations as well as contexts instead of just question-answering. In practice oriented papers mainly general application scenarios are described, e.g., assistants for customer communication [3], FAQ answering [e.g., 36, 59] as well as challenges or general conditions of chatbot applications [e.g., 29, 42]. Some papers also address e-commerce (aka conversational commerce) or customer self-services using chatbots, e.g., booking flights or banking [37, 42, 55]. Some legal aspects and challenges are described once [11]. Subsuming, we could identify 14 papers with an external enterprise focus on communicating with customers. However, scientific research results are missing and mostly general statements could be derived from practice-oriented papers. Nonetheless, those can point out an evidence for the need of chatbots.

Furthermore, we identified 11 relevant papers belonging to information acquisition. Most authors describe various concepts or prototypes - sometimes with evaluation results. In [48] a schema was derived that outlines, which communication patterns exist for information acquisition and how those patterns should be implemented in a chatbot. A general ontology-based chatbot was described by [1], which can easily be transferred between different subject areas and thereby reduces maintenance. In $[12,49,57,58]$ different conceptual approaches were described. In those cases, users can get various information, e.g., upcoming tasks or activities. Also a chatbot, which uses the Googlesearch engine as the backend was identified [47]. Queries are forwarded to Google and the result is fetched back and displayed in the dialog. Another concept for information acquisition uses a hybrid knowledge base of AIML (for permanent answers) and a database (for frequently changing answers), e.g., CRM or ERP systems [50]. In [56] a chatbot based on bigrams for similarity calculation and a relational database as data storage is described. Furthermore, in [32, 61] user satisfaction in search tasks with chatbots were analyzed. Summarizing, concepts or prototypes mostly focus this application area. However, requirements for this task are lacking as well as detailed evaluations of the resulting chatbots. Thus, detailed insights concerning developed concepts and implementations are also missing. Despite these aspects, this category receives the most attention in the scientific community.

For the field of self-service, the authors point out some application areas, like travel expense accounting or chatbots as personal accountants [18, 30]. Also chatbots can be used to change the master data of employees as well as retrieve remaining days off [27]. Overall, three papers address only abstract and general application areas without going into detail. We declare this by the practice-oriented focus combined with a lacking research method. Therefore, concepts or prototypes are missing and the field of self-service is unexplored. Scientific research is necessary to examine the application area in order to provide insights how to develop self-service chatbots.

Three articles examine the use of chatbots for education and training tasks. For instance, chatbots should support employees' onboarding processes by answering corresponding questions and help employees to learn company specifics [28]. In addition, lifelong learning at work can be addressed by this as well. Another two relevant papers describe a chatbot [21, 43], which can provide resources for learning via the natural dialog like an automated teaching assistant. In addition, it is possible to evaluate the user based on asked questions. Summing up, only a few contributions exist, which focus mainly on single concepts without outlining requirements or providing evaluation results.

In addition, we could identify only one paper which addresses the collaborative work [38]. The authors explain how chatbots can reduce friction by inappropriate tools in collaborative teamwork setting. They present some realizations, which can be used in communication tools like Slack. Overall, the paper points out the usage potential, but actual research about the use of chatbots for collaborative work is missing.

At last, most of our identified papers are without focus on a specific research topic. Papers in this category multiple aspects, which are described in the following. Some tackle the historical evolution of the technology or relevant components [e.g., 9, 53]. Furthermore, in practice-oriented papers, various general application areas, challenges or objectives were described [e.g., 20, 39]. In addition, three concepts of chatbots without a specified application area are provided. [2] present a chatbot with a 3D avatar and facial expressions in addition to the natural language dialog. Also [6] describe a chatbot which determines matchings based on examples instead of rules. In [60] a chatbot is described, which generates its knowledge base with the help of online available API documentations. After a preprocessing, the documenttation is accessible through the dialog. Also some contributions tackle the adaption of dialogs to enable inquiries when ambiguities occur [44] or to handle the user intention [45]. At last [66, 67] looked at user behavior, perception and expectations. To sum up, all of these papers deliver only sketchy insights in the research area. Nonetheless, they point out some application areas 
or approaches for using chatbots at digital workplaces, which have to be examined in detail.

\subsection{Potentials}

In addition, we examined actual potentials of using chatbots at the digital workplace mentioned in the analyzed literature (see Table 2). Since some authors address multiple application potentials of chatbots, it is necessary to record them independently of the research contribution (see section 4.2). In doing so, we also tried to gain detailed insights into the application areas as described in section 2.1. As seen in table 2, we identified four potentials. In addition, most of the papers discuss information search tasks in general. The papers of criterion 1 focus mostly on the answering of customer questions. Many of the detailed potentials (e.g., $\mathrm{P} 1_{3}-\mathrm{P} 1_{8}$ ) were only discussed in literature of criterion 2 and were therefore transferred by us to the digital workplace.

First of all, chatbots are able to support various kinds of information search tasks (P1) to provide user with needed information [e.g., 2, 50]. These can be for instance FAQs to relieve employees in the customer service by automating recurring questions [e.g., 14, 29]. Also general questions, e.g., external questions from customers as well as internal questions from employees, can be answered automatically by chatbots [e.g., 26, 56]. Furthermore, chatbots provide channels to get product information for purchase preparation [e.g., 9, 37]. In addition, different minor daily information can be retrieved via a chatbot, e.g., upcoming tasks, appointments or meetings [46]. Also it is possible to retrieve information during maintenance processes [68].

Secondly, chatbots are able of mapping standard routine processes $(\mathrm{P} 2)$ [e.g., 55, 66]. To do this, they guide employees step-by-step through processes, query necessary entries and perform corresponding resulting steps [e.g., 30, 54], e.g., master data changes or travel expense accounting [e.g., 27, 30]. Also employees can use chatbots to arrange meetings in a natural dialog. The chatbot negotiates between the participants and set up an appointment [e.g., 20, 36, 38].

In addition, as shown in section 4.2, chatbots can be used for teaching and learning tasks at the digital workplace (P3). Chatbots can teach learning content in a natural language dialog in such a way that employees can for example demand content for training at the workplace [e.g., 2, 43]. In addition, employees and their learning progress can be evaluated based on questions. Furthermore, recommendations for further learning steps for employees can be given by chatbots [4, 21].

\subsection{Objectives}

Lastly, we examined the mentioned objectives of using chatbots at digital workplaces (see Table 2).

Chatbots provide a natural language user interface to information systems (O1). This allows (enterprise) applications to be easily integrated without the user having to install additional software. The chatbot backend uses existing interfaces to access integrated (enterprise) applications or (web) services and provides them in the same communication channel. This will reduce media discontinuity and application overload within daily work routines [e.g., 5, 20, 38]. Instead of learning user interfaces, employees can execute processes or tasks intuitive and with natural language. This will also decrease frustration with existing applications [e.g., 3, 12].

Additionally, chatbots should provide an uniformly, device-independent and mobile access to application systems through the use of, e.g., messaging services as an interface (O2) [39, 54].

Furthermore, chatbots are supposed to increase efficiency and productivity of work by using speech and providing context information (O3) [e.g., 36, 66].

Also, decrease of time efforts $(\mathrm{O} 4)$ and reduced costs (O5) are objectives of using chatbot applications at the digital workplace, e.g., by automatically answering customer questions [e.g., 15, 53, 66].

Lastly, chatbots should relieve employees by taking over or automating tasks, e.g., customer service, so that employees can focus on complex or enterprise-relevant tasks (O6). For tasks that can not to be automated completely, chatbots should try to assist employees as much as possible [e.g., 27, 39, 42].

\section{Discussion of the results}

The results of our structured literature review indicate that research gaps exist in many of the outlined research areas belonging to the actual use of chatbots at digital workplaces. However, as shown in section 4.2, we could already identify six research areas targeting the use of chatbots. In the identified scenarios information acquisition and customer support are mainly addressed. In most papers, authors just describe specific concepts - only few are evaluated. Officerelated topics like collaborative work, education and learning or self-service are currently only addressed by few authors who mostly mention only sketchy application areas. Therefore, we conclude, that generalized statements for the design of chatbots for the digital workplace are not inferable because requirements as well as evaluations for the designed approaches are missing. 
Furthermore, we showed that the two main potentials of chatbots are information search tasks and standardized routine processes (see section 4.3). As shown in section 4.2, the information acquisition is already examined in some articles, e.g., with concepts or evaluations. For the support of standardized routine processes, only few limited results are available. In addition, chatbots should provide a natural language interface for enterprise applications, which is not addressed in research yet. Furthermore, many external application scenarios target at communication with customers are described. In contrast to that, internal application scenarios at the workplace are missing so far. This is also consistent with the identified research contributions, as the focus currently lies on customer support and information gathering, both of which are not purely company or office-workplace related tasks.

In the analysis of objectives (see section 4.4), we pointed out that firstly chatbots should integrate enterprise application systems in natural language dialogs. Secondly, chatbots should support employees by taking over or automating daily tasks. Both objectives correspond to the identified application areas. However, not a single paper addresses this fully by describing a concept or a prototype (see section 4.2). Thus, we conclude that there is also a research gap.

Table 3. Contributions to the design of chatbots

\begin{tabular}{|c|c|c|c|c|c|c|}
\hline & 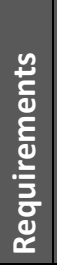 & 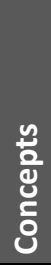 & 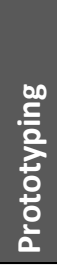 & $\frac{\text { 을 }}{\frac{\pi}{0}}$ & 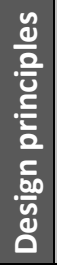 & $\begin{array}{l} \\
\text { む̀ } \\
\text { ¿ } \\
\text { ¿ } \\
\text { w }\end{array}$ \\
\hline Customer support & 0 & 4 & 1 & 3 & 0 & 14 \\
\hline Information acquisition & 0 & 8 & 7 & 5 & 1 & 11 \\
\hline Self-service & 0 & 0 & 0 & 0 & 0 & 3 \\
\hline Education and Training & 0 & 1 & 2 & 0 & 0 & 3 \\
\hline Collaborative work & 0 & 0 & 0 & 0 & 0 & 1 \\
\hline Without Focus & 1 & 6 & 2 & 5 & 0 & 20 \\
\hline Sum total & 1 & 19 & 12 & 13 & 1 & 52 \\
\hline
\end{tabular}

Furthermore, we analyzed the contributions to the design knowledge base. Since many of the contributions are from practice papers, insights in scientific publications are missing. Therefore, we analyzed the present design contributions to provide an entry point for future scientific studies. For this purpose, we examined whether the papers provide insights concerning requirements, concepts, artefacts, evaluations and generalized design principles [7, 25] (see Table 3). Clearly, we indicate that requirements as well as generalized results, e.g., design principles, for the design of chatbots at the digital workplace are missing. This is especially surprising as 19 papers describe concepts and 12 papers provide prototypes. However, in order to derive generalized results, it is necessary to identify design patterns for application areas. First, requirements for different application areas have to be identified in order to construct reproducible concepts and prototypes. Finally, it is necessary to evaluate them to derive valid design recommendations.

In addition, we showed in section 4 that practiceoriented insights are missing. Even though, we identified some practice-oriented papers, which only address general topics (like customer support). Mainly, the identified empirical research contributions focus on evaluations of the designed chatbots or underlying conditions. Currently there are no (comprehensive) empirical studies on the use of chatbots at the digital workplace. Therefore, a comprehensive analysis underpinned by practical insights of application areas and their requirements as well as general conditions is required. Especially since chatbots can currently only be used in limited and structured areas of responsibility or work $[1,29,55]$, it is necessary to examine them in detail. We delivered a first approach for this through our structured literature review Thus, we could show first literature-based results for the topic, but it is still necessary to survey practice-oriented findings in order to investigate and validate them in detail.

\section{Open research questions}

Overall, based on our findings and the discussion, we postulate the following open questions that should be targeted in future chatbot-related research at digital workplaces (see Table 4). Even though our questions are generally formulated, they can easily be applied to specific use cases. To describe them in detail, we adapt them in the following to the application area of information acquisition (see section 4.1).

Table 4. Open research questions Topics to address

Q1. Which application areas are viable for chatbots at the digital workplace?

Q2. Which prerequisites have to be considered?

Q3. Which factors inhibit the usage of chatbots?

Q4. Which factors support the usage of chatbots?

Q5. How should chatbots be designed?

Q6. What are the specific requirements?

Q7. What are the resulting benefits of the usage of chatbots?

Q8. What are design principles for chatbots?

Q9. What elements should a theory for chatbots at the workplace include? 
Firstly, as shown in section 4.2 most of the relevant papers focus on information acquisition mainly in the use case of customer support. As stated above, chatbots are currently not widely used at the workplace and we based our findings therefore on papers matching criterion 2. Therefore, a first question arose, if our application areas are the viable ones or if there are more application areas possible, which are not reflected in the literature currently (Q1). For the identified application areas, prerequisites must be surveyed (Q2). Extending this, it is necessary to investigate positive or negative factors (Q3-Q4), e.g., challenges, opportunities or objectives, which influence the adoption of chatbots at the workplace. Therefore, research is still necessary for the case of information acquisition in workplace context, e.g., chatbot access for enterprise (knowledge) databases or internal resources instead of predefined FAQs. Nonetheless, the same questions arose for all other application areas as identified in section 4.2, e.g., master data changes or travel expense accounting. Since we scrutinized only the literature belonging to the application areas and objectives, we recommend performing further practice-oriented behavioral studies to answer the open questions e.g., by interviewing experts or practitioners. In those studies, (potential) users should be surveyed to ascertain the application of chatbots in detail and in real-world scenarios. By answering these questions, theories of explanation and maybe also of prediction can be used [24].

Secondly, as shown in section 5, just a few contributions targeting the design of chatbots exist and most of them are concepts or prototypes. However, requirements as a prerequisite for the design of chatbots at the workplace are lacking currently. We suggest addressing the design of chatbots by design research (Q5). At first the viable application areas have to be derived and defined, e.g., the mentioned information acquisition. Following this, specific requirements for each application area must be identified (Q6; e.g., necessary database interfaces, organization of data maintenance, security measures as well as general requirements like input or output modality, NLP provider). Next, these requirements have to be transformed into (software) artifacts (Q5; e.g., a chatbot for answering employee question like "how can I change my password?" or "where can I find the documents for travel accounting?"). These artifacts have to be evaluated in further (empirical) studies, e.g., laboratory experiments to gain feedback. The results of the evaluation step can also be used to analyze impacts of using chatbots in enterprises (Q7). Finally, all of these results have to be converted into generalized design principles to address the whole design process (Q8). Hereby, the results of the individual design research can be adapted to different application areas. For instance, generalized results of an information acquisition chatbot can be used to design a chatbot for internal processes. By answering these further design research questions a theory of design and action can be addressed finally [24]. Since currently no specific theories for chatbots at the workplace are present, the question arose which components theories should have (Q9), e.g., to measure the effects of adaptions or the hindering factors. Existing theories (like the media-richness theory) can be used as a starting point for further theory development.

\section{Conclusion}

In this paper, we examined the state of the art of chatbots at the digital workplace. Therefore, we asked two research questions and answered them by conducting a structured literature review. As shown, only a few scientific findings exist, which tackle the usage of chatbots at digital workplaces especially for collaborative work between employees.

As in any research study, limitations need to be considered. We evaluated existing scientific and practice-oriented literature until end of 2017 , so there could be new published papers in the meantime. In addition, we included many articles of closely related topics, which do not mention digital workplaces directly (see Table 1 and Figure 3). Since there are only a few contributions matching criterion 1 , we used this approach to survey the state of the art of using chatbots at digital workplaces. Therefore, it is still necessary to gain practice insights in the subject area to validate and extend our findings. To address this, we recommend answering our postulated open questions.

\section{References}

[1] Al-Zubaide, H. and A.A. Issa, "OntBot: Ontology based chatbot", in 4th International Symposium on Innovation in Information \& Communication Technology, ISIICT, Amman. 2011.

[2] Angga, P.A., W.E. Fachri, A. Elevanita, Suryadi, and R.D. Agushinta, "Design of chatbot with 3D avatar, voice interface, and facial expression", in International Conference on Science in Information Technology, ICSITech, Yogyakarta. 2015.

[3] Aquino, J., "Virtual Assistants Enter The Enterprise", CRM Magazine, 16(12), 2012, pp. 30-34.

[4] Augello, A., G. Pilato, A. Machi, and S. Gaglio, "An Approach to Enhance Chatbot Semantic Power and Maintainability: Experiences within the FRASI Project", in IEEE Sixth International Conference on Semantic Computing, ICSC, Palermo, Italy. 2012.

[5] Bager, J., "Chatbot, mach mal!", c't - Magazin für Computertechnik, (12), 2016, p. 92. 
[6] Bang, J., H. Noh, Y. Kim, and G.G. Lee, "Example-based chat-oriented dialogue system with personalized long-term memory", in International Conference on Big Data and Smart Computing, BigComp, Jeju. 2015.

[7] Baskerville, R. and J. Pries-Heje, "Explanatory Design Theory", Business \& Information Systems Engineering, 2(5), 2010, pp. 271-282.

[8] Begau, K., R. Gabriel, F. Knittel, and H. Taday, "Büroinformations- und -kommunikationssysteme Aufgaben, Systeme, Anwendungen", in Arbeitsberichte des Lehrstuhls für Wirtschaftsinformatik der Ruhr-Universität Bochum, R. Gabriel, Editor. 1993: Bochum.

[9] Berg, M., "Natürlichsprachlichkeit in Dialogsystemen Stand der Technik in Industrie und Forschung", InformatikSpektrum, 36(4), 2013, pp. 371-381.

[10] Bott, G., "Digitale Sprachassistenten sind Neuland in Unternehmen", marconomy, 2017.

[11] Brunotte, N., "Virtuelle Assistenten: Europäische Union sieht Handlungsbedarf bei rechtlichen Rahmenbedingungen", Datenschutz-Berater, (7-8), 2017, p. 155.

[12] Carayannopoulos, S., "Using chatbots to aid transition", International Journal of Information and Learning Technology, 35(2), 2018, pp. 118-129.

[13] Chai, J., J. Lin, W. Zadrozny, Y. Ye, M. StysBudzikowska, V. Horvath, N. Kambhatla, and C. Wolf, "The Role of a Natural Language Conversational Interface in Online Sales: A Case Study", International Journal of Speech Technology, 4(3-4), 2001, pp. 285-295.

[14] Chakrabarti, C. and G.F. Luger, "A Semantic Architecture For Artificial Conversations", in Joint 6th International Conference on Soft Computing and Intelligent Systems and 13th International Symposium on Advanced Intelligence Systems, SCIS-ISIS, Kobe, 20-24 November. 2012.

[15] Chakrabarti, C. and G.F. Luger, "Artificial conversations for customer service chatter bots: Architecture, algorithms, and evaluation metrics", Expert Systems with Applications, 42(20), 2015, pp. 6878-6897.

[16] Cooper, H., "Organizing knowledge syntheses: A taxonomy of literature reviews", Knowledge in Society, 1(1), 1988, pp. 104-126.

[17] Daft, R.L., R.H. Lengel, and L.K. Trevino, "Message Equivocality, Media Selection, and Manager Performance: Implications for Information Systems", MIS Quarterly, 11(3), 1987, pp. 355-366.

[18] Dämon, K., "Wie Chatbots die Buchhaltung verändern", WirtschaftsWoche Online, 2017.

[19] Fettke, P., "State-of-the-Art des State-of-the-Art - Eine Untersuchung der Forschungsmethode "Review" innerhalb der Wirtschaftsinformatik - Forschungsmethodik, Metaanalyse, Forschungssynthese, Forschungsintegration, Forschungsbewertung, Wirtschaftsinformatik, 48(4), 2006, pp. 257-266.

[20] Følstad, A. and P.B. Brandtzæg, "Chatbots and the new world of HCI", interactions, 24(4), 2017, pp. 38-42.
[21] Fonte, F.A.M., J.C.B. Rial, and M.L. Nistal, "TQ-Bot: An AIML-based Tutor and Evaluator Bot", Jounal of Universal Computer Science, 15(7), 2009, pp. 1486-1495.

[22] Gentsch, P., Künstliche Intelligenz für Sales, Marketing und Service, Springer Fachmedien Wiesbaden, Wiesbaden, 2018.

[23] Gränig, A., C. Felden, and M. Piechocki, "Status Quo und Potenziale der eXtensible Business Reporting Language für die Wirtschaftsinformatik", Wirtschaftsinformatik, 53(4), 2011, pp. 225-234.

[24] Gregor, S., "The Nature of Theory in Information Systems", MIS Quarterly, 30(3), 2006, pp. 611-642.

[25] Gregor, S. and A.R. Hevner, "Positioning and Presenting Design Science Research for Maximum Impact", MIS Quarterly, 37(2), 2013, pp. 337-355.

[26] Grodzietzki, S.E., "Mein Bot und Ich - digitales Lernen, Arbeiten und Leben", Wissensmanagement, (6-7), 2017, pp. 18-19.

[27] Gyton, G. and R. Jeffsry, "These are the experts deciding the future of HR ... shouldn't you know who they are?", People Management, 2017, pp. 24-31.

[28] Han, V., "Are Chatbots the Future of TRAINING?", Talent Development, 71(9), 2017, pp. 42-46.

[29] Heckel, M. and S. Ermisch, "Hallo! Wie kann ich Ihnen heute helfen?", impulse, (7/8), 2017, pp. 76-77.

[30] Henrich, O., "Chatbots auf dem Vormarsch: Der künstlich-intelligente Buchhalter kommt", Wirtschaftsinformatik \& Management, (6), 2017, pp. 72-74.

[31] Hobert, S., Empirische Erkenntnisse und Gestaltungsansätze zum Einsatz von Wearable Computern im Industriesektor, Göttingen, 2018.

[32] Kiseleva, J., K. Williams, J. Jiang, A. Hassan Awadallah, A.C. Crook, I. Zitouni, and T. Anastasakos, "Understanding User Satisfaction with Intelligent Assistants", in Proceedings of the 2016 ACM on Conference on Human Information Interaction and Retrieval, D. Kelly, R. Capra, N. Belkin, J. Teevan, and P. Vakkari, Editors, CHIIR, Carrboro, North Carolina, USA. 2016.

[33] Klaffke, M., ed., Arbeitsplatz der Zukunft, Springer Fachmedien Wiesbaden, Wiesbaden, 2016.

[34] Klaffke, S. and S. Reinheimer, "Enterprise 2.0 Gestaltung der Büro-Informationsund Kommunikationstechnologie", in Arbeitsplatz der Zukunft, M. Klaffke, Editor. 2016. Springer Fachmedien Wiesbaden: Wiesbaden.

[35] Köffer, S., "Designing the digital workplace of the future - what scholars recommend to practitioners", in 36th International Conference on Information Systems, ICIS 2015, Fort Worth. 2015.

[36] Korenziowski, P., "Bots Should Be in Your Contact Center's Future: The technology emerges as businesses look to artificial intelligence to improve customer servic", CRM Magazine, 21(5), 2017, pp. 28-32.

[37] Kuhn, T., "Künstliche Intelligenz: Ich kann dich nicht verstehen", WirtschaftsWoche, (23), 2017, pp. 51-52. 
[38] Lebeuf, C., M.-A. Storey, and A. Zagalsky, "How Software Developers Mitigate Collaboration Friction with Chatbots", in Talking with Conversational Agents in Collaborative Action Workshop at the 20th ACM conference on Computer-Supported Cooperative Work and Social Computing, CSCW, Portland. 2017.

[39] Lebeuf, C., M.-A. Storey, and A. Zagalsky, "Software Bots", IEEE Software, 35(1), 2018, pp. 18-23.

[40] Lestarini, D., S.P. Raflesia, and K. Surendro, "A conceptual framework of engaged digital workplace diffusion", in 9th International Conference on Telecommunication Systems Services and Applications, TSSA 2015, Bandung. 2015.

[41] Mallios, S. and N. Bourbakis, "A survey on human machine dialogue systems", in 7th International Conference on Information, Intelligence, Systems \& Applications, IISA, Chalkidiki, Greece. 2016.

[42] Masterson, M., "Intelligent Virtual Assistants", Speech Technology Magazine, 20(1), 2015, pp. 35-37.

[43] Mikic, F.A., J.C. Burguillo, M. Llamas, D.A. Rodríguez, and E. Rodríguez, "Charlie : An AIML-based Chatterbot which Works as an Interface among INES and Humans", in EAEEIE Annual Conference, Valencia. 2009.

[44] Montero, C.A.S. and K. Araki, "Enhancing Computer Chat: Toward a Smooth User-Computer Interaction", in Knowledge-Based Intelligent Information and Engineering Systems (KES 2005), R. Khosla, R.J. Howlett, and L.C. Jain, Editors. 2005. Springer Berlin Heidelberg: Berlin, Heidelberg.

[45] Neves, A.M.M., F.A. Barros, and C. Hodges, "iAIML: a Mechanism to Treat Intentionality in AIML Chatterbots", in Proceedings of the 18th IEEE International Conference on Tools with Artificial Intelligence, ICTAI. 2006. IEEE Computer Society: Los Alamitos.

[46] Panser, F. https://www.marconomy.de/so-koennendigitale-sprachassistenten-das-business-modernisieren-a660770/, accessed 4-3-2018.

[47] Quarteroni, S. and S. Manandhar, "A Chatbot-based Interactive Question Answering System", in Proceedings of the 11th Workshop on the Semantics and Pragmatics of Dialogue, Trento. 2007.

[48] Radlinski, F. and N. Craswell, "A Theoretical Framework for Conversational Search", in Proceedings of the 2017 Conference on Conference Human Information Interaction and Retrieval, R. Nordlie, N. Pharo, L. Freund, B. Larsen, and D. Russel, Editors, CHIIR, Oslo. 2017.

[49] Ranoliya, B.R., N. Raghuwanshi, and S. Singh, "Chatbot for university related FAQs", in International Conference on Advances in Computing, Communications and Informatics (ICACCI), ICACCI, Udupi. 2017.

[50] Reshmi, S. and K. Balakrishnan, "Implementation of an inquisitive chatbot for database supported knowledge bases", Sadhana, 41(10), 2016, pp. 1173-1178.

[51] Richter, A., P. Heinrich, A. Stocker, and G. Schwabe, "Digital Work Design", Business \& Information Systems Engineering, 60(3), 2018, pp. 259-264.
[52] Russell, D.M., "Ubiquitous search for smart workspaces", Universal Access in the Information Society, 11(3), 2012, pp. 337-344.

[53] Satu, S., H. Parvez, and Shamim-Al-Mamun, "Review of integrated applications with AIML based chatbot", in 1st International Conference on Computer \& Information Engineering, ICCIE, Rajshahi. 2015.

[54] Schäffner, T., "Virtuelle Assistenten - was Chatbots künftig leisten", Wissensmanagement, (8), 2017, pp. 44-45.

[55] Schonschek, O., "Was Unternehmen über Chatbots wissen müssen", Computerwoche, (8-9), 2017, pp. 14-17.

[56] Setiaji, B. and F.W. Wibowo, "Chatbot Using a Knowledge in Database: Human-to-Machine Conversation Modeling", in 7th International Conference on Intelligent Systems, Modelling and Simulation, ISMS, Bangkok. 2016.

[57] Shawar, B.A., "Chatbots are natural web interface to information portals", in 6th International Conference on Informatics and Systems, INFOS, Kairo. 2008.

[58] Shawar, B.A., E. Atwell, and A. Roberts, "FAQChat as an Information Retrieval System", in Proceedings of the 2nd Language and Technology Conference, Posen. 2005.

[59] Strehlitz, M., "Der intelligente digitale Assistent", ITDirector, (11), 2017, pp. 30-32.

[60] Vaziri, M., L. Mandel, A. Shinnar, J. Siméon, and M. Hirzel, "Generating chat bots from web API specifications", in Proceedings of the 2017 ACM SIGPLAN International Symposium on New Ideas, New Paradigms, and Reflections on Programming and Software, Onward!, Vancouver. 2017.

[61] Vtyurina, A., D. Savenkov, E. Agichtein, and C.L.A. Clarke, "Exploring Conversational Search With Humans, Assistants, and Wizards", in Proceedings of the 2017 CHI Conference Extended Abstracts on Human Factors in Computing Systems, CHI EA, Denver. 2017.

[62] Wallace, R.S., "The Anatomy of A.L.I.C.E", in Parsing the Turing Test, R. Epstein, G. Roberts, and G. Beber, Editors. 2009. Springer Netherlands: Dordrecht.

[63] Webster, J. and R. Watson, "Analyzing the Past to Prepare for the Future: Writing a Literature Review", MIS Quarterly, 26(2), 2002, pp. 13-23.

[64] Weizenbaum, J., "ELIZA - A Computer Program For the Study of Natural Language Communication Between Man And Machine", Communications of the ACM, 9(1), 1966, pp. 36-45.

[65] White, M., "Digital workplaces: Vision and reality", Business Information Review, 29(4), 2012, pp. 205-214.

[66] Zamora, J., "I'm Sorry, Dave, I'm Afraid I Can't Do That: Chatbot Perception and Expectations", in Proceedings of the 5th International Conference on Human Agent Interaction, B. Wrede, Y. Nagai, T. Komatsu, M. Hanheide, and L. Natale, Editors, HAI'17, Bielefeld, Germany. 2017.

[67] Zamora, J., "Rise of the Chatbots: Finding a Place for Artificial Intelligence in India and US", in Proceedings of the 22nd International Conference on Intelligent User Interfaces Companion, IUI Companion, Limassol. 2017.

[68] Zirn, C. https://www.cio.de/a/chatbots-wasunternehmen-wissen-muessen,3563697, accessed 3-22-2018. 